\title{
A Novel Transparent UWB Antenna for Photovoltaic Solar Panel Integration and RF Energy Harvesting
}

Thomas Peter, Member, IEEE , Tharek Abd Rahman, Member, IEEE, S. W. Cheung, Senior Member, IEEE, Rajagopal Nilavalan, Senior Member, IEEE, Hattan F. Abutarboush, Member, IEEE, and Antonio Vilches, Member, IEEE

Abstract-A novel transparent ultra-wideband antenna for photovoltaic solar-panel integration and RF energy harvesting is pro-posed in this paper. Since the approval by the Federal Communications Committee (FCC) in 2002, much research has been undertaken on UWB technology, especially for wireless communications. However, in the last decade, UWB has also been proposed as a power harvester. In this paper, a transparent cone- top-tapered slot antenna covering the frequency range from 2.2 to $12.1 \mathrm{GHz}$ is de-signed and fabricated to provide UWB communications whilst integrated onto solar panels as well as harvest electromagnetic waves from free space and convert them into electrical energy. The antenna when sandwiched between an a-Si solar panel and glass is able to demonstrate a quasi omnidirectional pattern that is characteristic of a UWB. The antenna when connected to a $2.55-\mathrm{GHz}$ rectifier is able to produce $18-\mathrm{mV} d \mathrm{~d}$ in free space and 4.4-mV dc on glass for an input power of $10 \mathrm{dBm}$ at a distance of $5 \mathrm{~cm}$. Although the antenna presented in this paper is a UWB antenna, only an operating range of 2.49 to $2.58 \mathrm{GHz}$ for power scavenging is possible due to the limitation of the narrowband rectifier used for the study.

\section{INTRODUCTION}

TRANSPARENT antennas for communications have been researched on by only a dedicated few for the last two decades [1]-[9]. These antennas were either semitransparent like those with antenna geometries made of opaque conductive materials on a transparent substrate such as polymers or fully transparent such as those designed on transparent conductive oxides (TCOs) like ITO, AgHT, or PEDOT [10], [11]. The uniqueness of the latter type was the discreetness and the conformability they provided by virtue of the flexible nature of the substrate. In recent years, integration of solar cells with a trans-parent antenna was studied for harvesting energy from the sun

However, this was on a narrowband antenna that becomes directional as a result of the opaque solar cells or panel which tend to act as a reflector. This thus limits the potential of such an antenna for window glass applications. Similarly, introducing an opaque material like an amorphous-silicon (a-Si) solar panel with an aluminum back electrode will also make a ultra-wide-band (UWB) antenna directional.

However, in this paper, this is overcome through the design and development of a novel UWB antenna that can provide omnidirectionality even though integrated on an a-Si solar panel with a metal aluminum back electrode or ground. This enables the UWB antenna to transmit and receive signals wirelessly on both sides of the glass of buildings and windows of homes. This is the first time a transparent UWB antenna capable of maintaining an omnidirectional pattern on a solar panel is demonstrated.

The transparent UWB antenna, when connected to a rectifying circuit, serves also as a rectenna. A rectenna is a special type of antenna that is used to directly convert microwave energy into dc electricity. The idea was initially mooted by Nikola Tesla 100 years ago but experimentally researched, presented, and patented by Brown [13], [14]. It was Brown who first proposed the term rectenna as it consisted of 
an antenna combined with a rectifying circuit to convert radio or microwave power to dc power. Since then, numerous applications such as providing $d c$ power from space by receiving a microwave beam transmitted to earth by a geostationary solar-power satellite and converting it into dc power [15], wireless power transmissions in distributed wireless sensor networks [16], RFID tags [17], health monitoring [18], and most recently in Nokia mobile phones to provide standby power [19] have been demonstrated.

However, the applications in [16]-[19] harbor around small antennas that need to be small in size to keep the devices small and compact in line with the current trend towards small size, compact, and costeffective devices. In this paper, although a small compact transparent UWB cone-top-tapered slot antenna (CTSA) covering a frequency range from 2.2 to $12.1 \mathrm{GHz}$ is presented as a transceiver, solar harvester, and a rectenna, the application is not limited to small devices as the target destination is its use on glass windows/panels of buildings. The ample glass space provides a limitless area for large transparent wide-band antenna designs or UWB array implementation to harvest radio waves across a wide range of frequencies and provide strong gain "last mile" connection for the futuristic UWB -overfiber (UOF) network [20]. Such antennas on the glass of buildings besides providing wireless communications and allowing harvesting of solar energy can also scavenge relatively large amounts of power from signals coming from miles away. Incorporating the rectifier circuit as an integral part of the antenna as in [21] could make it even more cost effective and efficient. Using enhanced rectifier circuits that are currently under study could facilitate the simultaneous reception of data and con-version to dc power.

A basic model of two antennas combined using a Wilkinson power combiner can be used to explain how simultaneous power transmission and data transfer can be achieved. A Wilkinson power combiner is a split-T combiner with a resistive element placed between the two input arms, which has all three ports matched and at the same time provides isolation between the two input ports. Inequalities between the antenna elements in the array, due either to tolerance effects or to phase differences in the received signals, will cause a voltage difference to occur across the resistor and thus make a current flow through it [23]. This current is dissipated by the resistor, thus providing isolation between the antenna elements. By replacing the resistor with a rectifier circuit, the inequalities in the received signals may be used for power conversion while maintaining the isolation be-tween the antenna elements [23]. The dc power output will be larger for larger inequalities between the antenna elements. The inequality between the signals at the combiner input ports can be achieved by differing the lengths of the transmission lines from the antennas to the combiner input ports [23]. Hence, by moving the rectifier from the port at the bottom of the feed line and connecting it between the input arms, the feed line port is freed for data transmission. With the input of the rectifier connected to the combiner input arms, dc power can be tapped through a load at its output. However, with glass space being amply available, the use of dedicated antenna arrays to provide for power scavenging and data transmission separately would not be a constraint.

In this paper, a UWB CTSA is demonstrated as part of a UWB transceiver laminated on an a-Si solar panel, and as a rectenna both in free space and on glass to provide wireless communication and harvest solar and RF energy. The antenna integrated solar panel and rectenna designs are presented in Section II. The measurement setup for each application is elaborated further in Section III. The measurement results and analysis are presented in Section IV with the conclusion on the findings in Section V. 


\section{Antenna Design}

The geometry of the proposed transparent antenna is shown in Fig. 1(a). The antenna is designed as a coplanar-waveguide (CPW) fed cone-top-tapered slot antenna inscribed on a thin TCO polymer film, AgHT-4, with an overall size of $\mathrm{W} \times \mathrm{L}-17 \times 335 \mathrm{~mm}$. The feed gap $g$ is $0.1 \mathrm{~mm}$, and the feed line is de-signed to slowly taper and merge into the radiator. The antenna is first studied laminated on a 2-mm-thick glass, and later sandwiched between the 2-mm-thick glass and an a-Si solar panel. The antenna is designed and studied using computer simulation with the optimized dimension listed in Table I. The antenna size should normally be smaller when fabricated on glass with a higher dielectric constant, which is 5.7 for the 2-mmthick glass. However, this is not the case here because the thickness of the glass increases the size of the antenna. Hence, in the sandwich con figuration, the overall thickness of the glass is twice as that in the substrate configuration, resulting in a much bigger volume due to the size of the antenna as well as the thickness of the glass. In addition, it needs to be pointed out that the optimized dimensions of the antenna on glass are the same, whether it is laminated in the substrate or superstrate configuration. This is because the substrate base of the AgHT- 4 film PET is very thin and, as such, has a minimal effect on the radiation energy in the superstrate configuration. A thicker PET in the superstrate con-figuration, on the other hand, would have made the antenna appear sandwiched between the PET and glass, causing it to give a different result to that of a substrate configuration. The CTSA is fabricated as shown in Fig. 1(b) for measurement.

The AgHT-4, a layer of silver sandwiched between two layers of tin oxide and coated onto a polyethylene terephthalate (PET) base, has a surface resistivity of $4 \Omega$-square, a conductivity of $250000 \mathrm{~S} / \mathrm{m}$ and a thickness of $0.175 \mathrm{~mm}$, making it suitable for use on window glass of homes and buildings. It has a visible light transmittance (VBLT) of $75 \%$. This metallized polymer is commercially used as a sun-shielding film to cut down heat and conserve energy in homes and buildings.

\section{Antenna Design for Solar Integration}

For solar harvesting applications, the CTSA is sandwiched between a 2-mm glass superstrate representing a glass window and an a-Si solar panel (model: QF 8368 ) of $38 \times 86 \mathrm{~mm}_{2}$, as shown in the simulated structural view in Fig. 2. The a-Si solar cell has several layers, as shown in Fig. 3. However, in computer simulation, the p-type and n-type layers are not included due to the difficulty in accurately modelling them. The solar panel as per manufacturer is designed to work in low light and cloudy conditions.

\section{Rectenna Design}

For rectifying the electromagnetic (EM) signals in the rectenna, a narrowband rectifier designed in [24] is used in our studies, since it was the only handy rectifier available in our laboratory at the time of this work. However, the authors would like to highlight at this point that the CTSA in actual fact can be used as a broadband rectenna in conjunction with

broadband rectifier and that a narrowband rectifier was used only to demonstrate in principle the CTSA's RF harvesting capability. The Microwave Office (MWO) circuit schematic of the rectifier used for simulation is shown in Fig. 4, which is a double-diode (HSMS-2850) rectifier designed to work at a frequency of $2.55 \mathrm{GHz}$ and fabricated on a FR-4 microstrip board with a thickness of $1.6 \mathrm{~mm}$ and a 
dielectric constant of 4.2. The package inductance and capacitance are also modeled in the circuit schematic to give better accuracy. The voltage-doubler configuration used in the design helps improve the voltage sensitivity [24]. In Fig. 4, the rectifier circuit receives the EM energy from the antenna and converts it to dc power. The prototype of the integrated antenna-rectifier module or rectenna is shown in Fig. 5. In practical applications, the rectifier could be attached to a window sill and connected to the antenna using a transparent feed line to maintain transparency. Alternatively, a transparent rectifier circuit could be designed and integrated

onto the antenna with only the diodes and capacitors being noticeable, but this is left to future works.

\section{MEASUREMENT SETUP}

\section{Solar Energy}

A prototype of the CTSA sandwiched between a 2-mm glass superstrate and the a- Si solar panel is fabricated and measured for gain and radiation pattern using the Satimo StarLab system. Also, the voltage output of the solar panel is taken in free space, laminated with the antenna, and with the antenna sandwiched between the solar panel and the 2-mm-thick glass. This was carried out to see whether AgHT-4's (VBLT) of 75\% and the glass thickness affected the performance of the a -Si solar panel. The solar panel has a conversion rate of more than $15 \%$ and is able to deliver a voltage of $5.5 \mathrm{~V}$ and a current of $70 \mathrm{~mA}$ as per the manufacturer's specification in free space. An additional voltage output measurement for the solar panel with the prototype connected to a vector network analyzer (VNA) is also taken to see whether EM transmission in any way affects the performance of the solar panel. The measurements are done in the ambient light of fluorescence lighting as well as outdoor in the sun.

\section{RF Energy}

A diagrammatic representation of the experimental setup used for measuring the amount of RF power converted to dc power by the rectenna in free space is shown in Fig. 6. In Fig. 6, single-tone RF signal is generated using a signal generator and fed to a horn antenna. The rectenna placed at a certain distance is used to collect the RF signal transmitted from the horn and convert it to dc power. The amount of dc converted is used to measure the performance of the rectenna.

The return loss for a 30- $\mathrm{dBm}$ input from an RF signal source with the rectifier output terminated with a 50 $\Omega$ load is shown in Fig. 7. It can be seen that the recti fier has a maximum return loss of $25 \mathrm{~dB}$ at around $2.55 \mathrm{GHz}$, which is the frequency at which the rectifier has the maximum voltage sensitivity and optimum performance. This is further verified by feeding a signal with frequency from 2.5 to $2.6 \mathrm{GHz}$ at a step of $0.01 \mathrm{GHz}$ to the rectifier input with the output terminated with a $50-\Omega$ load resistor and recording the output voltage at each step. The results are listed in Table II, which indicates a maximum output of $25.6 \mathrm{mV}$ at $2.55 \mathrm{GHz}$. However, it should be noted from Fig. 7 that the rectifier, with a load of $50 \Omega$, has a 10-dB operating bandwidth from 2.49 to $2.58 \mathrm{GHz}$; thus, when integrated with an antenna, the rectifier should be able to convert RF signals in this operating range to dc power. This is evident from [25], since although the loop antenna presented there has a narrow 10-dB operating bandwidth of 900-952 MHz, the rectifier is still able to receive and rectify electromagnetic waves in the neighboring frequencies within the 10-dB operating bandwidth of the recti-fier from 785 to $1150 \mathrm{MHz}$. 
With the CTSA connected to the rectifier output, the return loss of the rectenna is measured again with results also shown in Fig. 7 for comparison. It can be seen that the return loss is max-imum at $2.53 \mathrm{GHz}$ due to a mismatch. This is expected since an available rectifier in the laboratory was used for the study, rather than one that was specifically designed to impedance match the antenna.

The horn antenna used in our studies shown in Fig. 6 has an average gain of $9.8 \mathrm{~dB}$ for the range 2.5 to $2.6 \mathrm{GHz}$. To deter-mine the optimum operating frequency of the CTSA rectenna, the horn antenna is connected to a signal generator to serve as a transmit antenna. The CTSA rectenna is then placed at the receiver end $5 \mathrm{~cm}$ away to receive the signal, as shown in Fig. 6. A 50- $\Omega$ load is connected to the rectenna output to tap the rec-tified dc. The output voltages at steps of $0.01 \mathrm{GHz}$ for a signal frequency sweep from 2.5 to $2.6 \mathrm{GHz}$ are shown in Table III. It can be seen that the CTSA rectenna has a maximum output of $9.4 \mathrm{mV}$ at $2.53 \mathrm{GHz}$. This confirms the system has an optimum operating frequency of $2.53 \mathrm{GHz}$ for a $50-\Omega$ load. More measurements have been done with a $100-\Omega$ load with results also shown in Table III, indicating that the optimum operating frequency shifts to $2.51 \mathrm{GHz}$ with a maximum output voltage of $18.0 \mathrm{mV}$, higher than the case with a $50-\Omega$ load.

\section{RESULTS AND ANALYSIS}

\section{Solar Energy}

The simulated and measured return losses of the CTSA in free space, laminated on the a-Si solar panel and sandwiched be-tween the solar panel and 2 - $\mathrm{mm}$ glass superstrate are all shown in Fig. 8 for comparison. The discrepancy between the simulated and measured return losses in free space is mainly attributed to the accuracy of the parameters used to model the AgHT-4 material in simulation and fabrication accuracy of the prototype. Additional discrepancies between the simulated and measured return losses when the CTSA is laminated on the a- Si solar panel, and when sandwiched between the 2 $\mathrm{mm}$ glass, is attributed to the accuracy of the parameters used to model the a-Si solar panel and the uncertain air gaps between the CTSA, a-Si solar panel, and 2-mm glass.

The overall simulated and measured realized peak gains of the CTSA in free space, laminated on the a-Si solar panel and sandwiched between a-Si solar panel and the glass superstrate are shown in Fig. 9. The antenna has quite low gains due to the high resistance of the AgHT-4 materials. The measured gain on an average varies from 3 to $5 \mathrm{~dB}$ lesser than that of free space. When the antenna in free space and laminated on the a-Si solar panel, the simulated and measured gains agree well. When the antenna is sandwiched between the solar panel and glass superstrate, the simulated and measured results also agree well at frequencies below $5 \mathrm{GHz}$. However, a large discrepancy occurs at higher frequencies, probably due to the uncertain air gap on both sides.

The measured radiation patterns in the $X-Y$ plane at different frequencies are depicted in Fig. 10. The measured radiation pat-terns for the sandwich con figuration are seen to be quasi-omnidirectional for all the four different frequencies tested at 3,5, 7, and $10 \mathrm{GHz}$. However, the results are quite encouraging, as this allows wireless communication access inside and outside the building when the solar panel integrated antenna module is mounted onto a glass window or glass building. Additional endeavours, of course, are 
needed to further improve the gain to enhance transmission range and one way of doing this is through arrays.

Table IV gives the voltage output obtained from the a- Si solar panel in the ambient light of the fluorescence lighting of a room and under the sun. A 5-K $\Omega$ load resistor was used so that the voltage output would be large enough for comparison in the different conditions.

A drop of $40 \%$ in voltage output is observed for the integrated module of the CTSA sandwiched between the glass and solar panel when compared with the voltage output of the solar panel only. In addition, the energy harvesting performance of the module is not affected in any way when it was connected to the VNA to measure the return loss. The low output in the sun is because the solar panel is designed to operate in poor lighting conditions.

\section{RF Energy}

Rectenna in Free Space: The solar energy harvested may not be dependent on the antenna, but the RF energy is. The gain of the antenna at which the rectifier is designed needs to be high to aid the RF to dc conversion of the EM waves harvested.

The rectenna's efficiency, $\eta_{\text {textennia }}$ for free space and on glass, is given by

$$
\eta_{\text {rectenna }}=\eta_{\text {rectifier }} \times \eta_{\text {antenna }}
$$

From Fig. 6, by knowing the transmitted power $\left(\mathrm{P}_{\mathrm{N}}\right)$, gain of the transmitter $\left(\mathrm{G}_{1}\right)$ and measuring the distance (d) between the horn and the antenna under test (AUT) and the dc rectified power ( $P$ cc) and calculating the received RF power ( $\Gamma_{a c}$ ) by the antenna, we are able to determine the rectenna efficiency from to (4) given as follows, where Aeff is the effective antenna aperture:

$$
\begin{aligned}
\mathbf{P}_{\mathrm{ac}} & =\frac{\boldsymbol{P}_{\boldsymbol{T}} \cdot \boldsymbol{G}_{\boldsymbol{T}}}{4 \boldsymbol{\pi} \boldsymbol{d}^{2}} \times \mathbf{A}_{\mathrm{eff}} \\
& =\frac{\boldsymbol{P}_{\boldsymbol{T}} \cdot \boldsymbol{G}_{\boldsymbol{T}}}{4 \boldsymbol{\pi} \boldsymbol{d}^{2}} \times \frac{\boldsymbol{G}_{\boldsymbol{R}} \boldsymbol{x} \boldsymbol{\lambda}^{2}}{4 \boldsymbol{\pi}} \\
\mathbf{P}_{\mathrm{dc}} & =\mathbf{P}_{\mathrm{ac}}=\times \boldsymbol{\eta}_{\text {rectifier }}
\end{aligned}
$$

Therefore,

$$
\text { rectifier efficiency, } \boldsymbol{\eta}_{\text {rectifier }}=\mathbf{P}_{\mathrm{dc} /} \mathbf{P}_{\mathrm{ac}} \text {. }
$$

Hence from (1),

$$
\text { rectenna efficiency }=\mathbf{P}_{\mathrm{dc} /} \mathbf{P}_{\mathrm{ac} X} \boldsymbol{\eta}_{\text {antenna }} .
$$


A maximum rectifier efficiency of $49 \%$ is observed over a $100-\Omega$ load for a $0-\mathrm{dBm}$ input power at $2.51 \mathrm{GHz}$. The rectenna system efficiency is determined by taking the output power for varying power inputs from $30 \mathrm{dBm}(0.001 \mathrm{~mW})$ to $0 \mathrm{dBm}(1 \mathrm{~mW})$. The measured output powers and efficiencies of the rectenna at the frequencies of 2.51 and $2.53 \mathrm{GHz}$ and with $50-\Omega$ and $100-\Omega$ loads for different input power levels in free space are shown in Figs. 11 and 12. It can be seen in Fig. 11 that there is no output power if the input power is below about $15 \mathrm{dBm}$, which is due to the requirement of power to turn on the rectifying diodes. The output powers for an $100-\Omega$ load are higher than those for a $50-\Omega$ load, which agree with the results of Table III. The measured system efficiency in Fig. 12 increases with load but at the different operating frequencies as earlier pointed out in Table III. Although the efficiency in Fig. 12 is notably low, this could be improved via arrays and also larger antennas or redesigning the geometry to have better gain at the desired frequency of operation.

Rectenna on Glass: The same design of the CTSA on glass takes on a slightly larger or smaller size, depending on the dielectric constant and thickness of the glass used. A higher dielectric constant could further improve the gain. However, a higher thickness could possibly attenuate the received signal and consequently deteriorate the efficiency of the system. Nevertheless, the dimensions of the antenna design should be optimized to obtain the maximum gain possible at the frequency of operation.

The optimized size of the CTSA for use on a 2-mm-thick glass with a dielectric constant of 5.7 is given in Table I. In our design, the CTSA on-glass is only $0.8 \%$ bigger than that of free space. The simulated and measured return losses on glass are presented in Fig. 13. The difference between the simulated and measured return losses here are attributed to fabrication accuracy and the uncertain air gap between the CTSA and 2-mm glass.

The voltage output for the CTSA on a 2-mm glass is shown in Fig. 14. The voltage output is optimum at $2.57 \mathrm{GHz}$. The lower voltage output for the CTSA on glass is attributed to the lower gain at $2.57 \mathrm{GHz}$ in comparison to that of the CTSA in free space as shown in Fig. 15. Hence, at frequencies between 6-7 and 8-9 GHz, higher voltage output can be expected.

Comparison With Existing Rectenna Solutions: A brief comparison with existing narrowband and broadband solutions published so far is presented to show that the CTSA rectenna is comparable as well as has several advantages. The most important consideration for a rectenna design is how to obtain high efficiency. This is usually done in two ways: i) to collect and deliver maximum power to the rectifying diode, and ii) to acquire high rectifier conversion efficiency through suppression of harmonics generated by the diode which reradiate from the antenna as power lost. To maximize the delivered power, antennas arrays [26]-[29], broadband and UWB antennas [30]-[35], and circularly polarized (CP) antennas [36], [37] have been built to increase conversion efficiency. The antenna arrays increase incident power delivered to the rectifier diode by enlarging antenna aperture and increasing antenna gain, while the broadband and UWB antennas enable relatively high RF power to be collectively received from various sources in their frequency range. CP antennas meanwhile offer better power reception through less polarization mismatch. Harmonic suppression has been achieved by way of introducing, microwave components such as a low pass filter (LPF) between the antenna and diode as well as on the dc output side of the diode, and using optimized rectenna design that has harmonic rejection capabilities, thus eliminating LPFs [38], [39]. 
The rectifying circuit of the proposed CTSA rectenna has LPFs incorporated, thus enabling it to suppress harmonics generated by the diodes. Although RF harvesting between 2.49 and $2.58 \mathrm{GHz}$ only is demonstrated here, the CTSA as a UWB antenna is capable of harvesting at various frequencies within its bandwidth range. The CTSA is smaller in size compared with those in [26]-[39] and has additional advantages as it can scavenge on both sides of the antenna due its omnidirectional characteristic and be aesthetically fitted on to glass panels of windows and buildings. Moreover, using a UWB antenna, the aperture is kept constant at higher frequencies compared with narrow-band patch antennas that would shrink in size with an increase in frequency.

The performance of the rectenna in terms of conversion efficiency is comparable with those previously reported [25], [36], The conversion efficiency of $49 \%$ that was achieved for the rectifier in this study over a $100-\Omega$ load could be higher if a larger load resistance is used. Conversion efficiency is dependent on the load resistor since, Pdc in (5) when expanded is

$$
\begin{aligned}
\boldsymbol{P}_{\boldsymbol{\alpha} c} & =\frac{\boldsymbol{V}_{\boldsymbol{d} c}^{2}}{\boldsymbol{R}_{\boldsymbol{L}}} \\
& =\boldsymbol{I}_{\boldsymbol{\alpha}}^{2} \times \boldsymbol{R}_{\boldsymbol{L}}
\end{aligned}
$$

where Vck is the voltage observed at the load resistor and RL and Ick is the rectified dc current that flows into the load resistor. Hence from (7), the higher the load resistor used, the higher the rectified dc power and, consequently, the higher the rectifier efficiency.

The only limitations seen with the CTSA is the very short distance of $5 \mathrm{~cm}$ over which it could be used to harvest RF energy. It is comparatively low compared with the next highest distances achieved of 40 [40] and $57 \mathrm{~cm}$ [24] with slightly bigger narrowband antennas of $40 \times 40 \mathrm{~mm}_{2}$ [41] and $30 \times 30 \mathrm{~mm}_{2}$ [24], respectively, at similar operating frequencies. This mainly stems from the low gain of the antenna, which is a resultant of the low conductivity and high loss of the AgHT -4 material. This could be overcome in future by using a high gain and highly trans-parent nanotechnology TCO, which is currently under development at the laboratory of our industrial collaborators [41].

Fidelity Factor: The fidelity factor (FF) measures the distortion produced by an antenna when radiating an input signal. The FF of the CTSA in free space, on the 2-mm glass, and sandwiched between the a-Si solar panel and 2-mm glass is shown in Fig. 16. Only simulated results are shown here as the VNA used in the studies had limited measurement options.

The time-domain response to calculate the FF was obtained by using the reference and received signal. A simulated 7.5-GHz bandwidth Gaussian signal centred at $6.85 \mathrm{GHz}$ was applied as the reference signal at the input port and a virtual probe was placed at a distance of $300 \mathrm{~mm}$ from the antennas. The FF of the CTSAs in free space $=08802$, on 2-mm glass $=0.9273$, and sandwiched between solar panel and 2-mm glass $=09001$. The FFs obtained indicate good correlation between the normalized input and transmitted signals in simulation. 


\section{CONCLUSION}

A novel transparent UWB CTSA that can maintain its omnidirectionality when integrated onto solar panels as well as harvest RF energy, besides being able also to provide wireless communication, has been presented. This makes the CTSA excellent for on-glass applications as it can provide a 3-in-1 solution in providing sun shielding from harmful UV rays, wireless communication, and energy harvesting. The capability of the antenna in delivering a quasi-omnidirectional pattern even though sandwiched between an opaque a-Si solar panel and glass makes it able to provide wireless transmission and reception on both sides of a glass window or glass building. Finally, the work presented in this paper lends weight and credibility to the fact that transparent antennas like those made from AgHT or even other TCOs when utilized on glass windows could potentially also be-come the new "last mile" connection for nextgeneration wire-less communications via UOF. 


\section{REFERENCES}

[1] R. N. Simons and R. Q. Lee, "Feasibility study of opti-cally transparent microstrip patch antenna," presented at the IEEE/AP-S/U.R.S.I Int. Symp. Radio Sci. Meet., Montreal, QC, Canada, Jul. 13-18, 1997.

[2] C. Mias, C. Tsakonas, N. Prountzos, D. C. Koutsogeorgis, S. C. Liew, C. Oswald, R. Ranson, W. M. Cranton, and C. B. Thomas, "Opti-cally transparent microstrip antennas," Antennas for Automotives (Ref. No.2000/002), IEE Colloq., pp. 8/1-8/6, 2000.

[3] J. A. Nessel, A. Zaman, R. Q. Lee, and K. Lambert, "Demonstration of an X-band multilayer Yagilike microstrip patch antenna with high directivity and large bandwidth," in Proc. IEEE Antennas Propag. Soc. Symp., , 2005, vol. 1B, pp. 227-230.

[4] N. Guan, H. Furuya, D. Delaune, and K. Ito, "Antennas made of transparent conductive films," in Proc. PIERS, China, Mar. 2008, p. 720.

[5] A. Katsounaros, Y. Hao, N. Collings, and W. A. Crossland, "Optically transparent antenna for ultra wide-band applications," in Proc. 3rd Eur. Conf. Antennas Propag. (EuCAP), Mar. 23-27, 2009, pp. 1918-1921.

[6] N. J. Kirsch, N. A. Vacirca, E. E. Plowman, T. P. Kurzweg, A. K. Fontecchio, and K. R. Dandekar, "Optically transparent conductive polymer RFID meandering dipole antenna," in Proc. IEEE Int. Conf. RFID, Apr. 27-28, 2009, pp. 278-282.

[7] T. Peter and R. Nilavalan, "Study on the performance deterioration of flexible UWB antennas," in Proc. Antennas Propag. Conf. Loughbor-ough (LAPC), 2009, pp. 669-672.

[8] T. Peter, R. Nilavan, H. F. AbuTarboush, and S. W. Cheung, "A novel technique and soldering method to improve performance of transparent polymer antennas," IEEE Antennas Wireless Propag. Lett., vol. 9, pp. 918-921, 2010.

[9] T. Peter, T. I. Yuk, R. Nilavalan, and S. W. Cheung, "A novel tech-nique to improve gain in transparent UWB antennas," presented at the Antennas Propag. Conf. Loughborough (LAPC), Nov. 2011.

[10] T. Yasin, R. Baktur, and C. Furse, "A comparative study on two types of transparent patch antennas," in Proc. XXXth URSI Gen. Assembly Scientif. Symp., Aug. 13-20, 2011, pp. 1-4.

[11] "Transparent Flexible Conductive Polymer Antennas" [On-line]. Available: http://www.ece.drexel.edu/opticslab/posters/Re-searchDay09_CondPoly.pdf

[12] S. V. Shynu, M. J. R. Ons, P. McEvoy, M. J. Ammann, S. J. McCor-mack, and B. Norton, "Integration of microstrip patch antenna with polycrystalline silicon solar cell," IEEE Trans. Antennas Propag., vol. 57, no. 12, pp. 3969-3972, Dec. 2009.

[13] W. C. Brown, "The history of power transmission by radio waves," IEEE Trans. Microw. Theory Tech., vol. 32, no. 9, pp. 1230-1242, Sep. 1984.

[14] W. C. Brown, "Microwave to DC converter," U.S. 3434 678, Mar. 25, 1969.

[15] W. C. Brown, "Status of the microwave power transmission compo-nents for the solar power satellite," IEEE Trans. Microw. Theory Tech., vol. MTT-29, no. 12, pp. 1319-1327, Dec. 1981.

[16] K. M. Z. Shams and M. Ali, "Wireless power transmission to a buried sensor in concrete," IEEE Sensor J., vol. 7, pp. 1573-1577, 2007.

[17] B. Strassner and K. Chang, "Passive $5.8 \mathrm{GHz}$ radio frequency identifi-cation tag for monitoring oil drill pipe," IEEE Trans. Microw. Theory Tech., vol. 51, no. 2, pp. 356-363, Feb. 2003.

[18] V. Sakamuri and J. Frolik, "Design of a $2.4 \mathrm{GHz}$ interrogator for a rectenna-based sensor system," in Proc. IEEE 12th Annu. Wireless Mi-crow. Technol. Conf. (WAMICON), Apr. 18-19, 2011, pp. 1-4.

[19] D. G. Rowe, "Nokia developing phone that recharges itself without mains electricity," The Guardian, Jun. 10, 2009 [Online]. Available: www.guardian.co.uk 
[20] X. Xing, Z. Enbo, L. Yu, T. I. Yuk, K. S. Lui, and K. K. Y. Wong, "Power-efficient photonic BPSK coded ultrawideband signal genera-tion," in Proc. Opt. Fiber Commun./Nat. Fiber Optic Eng. Conf. and Expo. (OFC/NFOEC), Conf., Mar. 6-10, 2011, pp. 1-3.

[21] T.-U. Hong, K.-M. Oh, H.-W. Lee, H. Nam, T.-S. Yun, D.-S. Lee, H.-I. Hwang, and J.-C. Lee, "Novel broadband rectenna using printed monopole antenna and harmonic-suppressed stub filter," Microw. Opt. Technol. Lett., vol. 52, pp. 1194-1197, 2010.

[22] H. J. Visser and R. J. M. Vullers, "Design equations for small loop-based rectennas," presented at the Antennas Propag. Conf. Loughbor-ough (LAPC), Nov. 2011.

[23] H. J. Visser, Approximate Antenna Analysis for CAD. Chichester, U.K.: Wiley, 2009.

[24] K. W. Lui, A. Vilches, and C. Toumazou, "Ultra-efficient microwave harvesting system for battery-less micropower microcontroller plat-form," IET Microw., Antennas, Propag., vol. 5, no. 7, pp. 811-817, May 2011.

[25] R. H. Chen, Y. C. Lee, and J. S. Sun, "Design and experiment of a loop rectenna for RFID wireless power transmission and data communica-tion applications," in Proc. PIERS, Beijing, China, Mar. 23-27, 2009,

pp. 528-531.

[26] N. Shinohara and H. Matsumoto, "Experimental study of large rectenna array for miceowave energy transmission," IEEE Trans. Microw. Theory Tech., vol. 46, pp. 261-268, Mar. 1998.

[27] Y. J. Ren and K. Chang, "5.8-GHz circularly polarized dual-diode rectenna and rectenna array for microwave power transmission," IEEE Trans. Microw. Theory Tech., vol. 54, no. 4, pp. 1495-1502, 2006.

[28] S. Dasgupta, B. Gupta, and H. Saha, "Development of circular mi-crostrip patch antenna array for rectenna application," presented at the IEEE INDICON 2010, Kolkata, India, Dec. 17-19, 2010.

[29] W. Huang, B. Zhang, X. Chen, K.-M. Huang, and C.-J. Liu, "Study on an s-band rectenna array for wireless microwave power transmission," Progr. Electromagn. Res., vol. 135, pp. 747-758, 2013.

[30] J. A. Hagerty and Z. Popovic, "An experimental and theoretical charac-terization of a broadband arbitrarily-polarized rectenna array," in Proc. IEEE MTT-S Int. Microw. Symp. Dig., Phoenix, AZ, USA, Jun. 2001, vol. 3, pp. 1855-1858.

[31] A. Slavova and A. S. Omar, "Wideband rectenna for energy recycling," in Proc. IEEE AP-S Int. Symp. Dig., Columbus, OH, USA, Jun. 2003,

pp. 954-957.

[32] D. Bouchouicha, F. Dupont, M. Latrach, and L. Ventura, "Ambient RF energy harvesting," presented at the Int. Conf. Renew. Energies Power Qual., Granada, Spain, 2010.

[33] J. A. Hagerty, N. D. Lopez, B. Popovic, and Z. Popovic, "Broadband rectenna arrays for randomly polarized incident waves," in Proc. 30th Eur. Microw. Conf., Oct. 2000, pp. 1-4.

[34] D. Bouchouicha, F. Dupont, M. Latrach, and L. Ventura, "Ambient RF energy harvesting," in Proc. Int. Conf. Renew. Energies Power Qual., Spain, Mar. 2010, pp. 1-5.

[35] C. M. Ghiglino, "Ultra-wideband (UWB) rectenna design for elec-tromagnetic energy harvesting," Ph.D. dissertation, Theory of Signal Commun. Dept., Telecommunications Technology Centre of Cat-alonia, Pontifical Catholic Univ. of Peru, Lima, Peru, 2010.

[36] J. Heikkinen and M. Kivikoski, "A novel dual-frequency circularly po-larized rectenna," IEEE Antennas Wireless Propag. Lett., vol. 2, pp. 330-333, 2003.

[37] B. Strassner and K. Chang, "5.8-GHz circularly polarized dual-rhombic-loop traveling-wave rectifying antenna for low power-den-sity wireless power transmission applications," IEEE Trans. Microw. Theory Tech., vol. 51, pp. 1548-1553, May 2003.

[38] A. Georgiadis, G. Andia, and A. Collado, "Rectenna design and optimization using reciprocity theory and harmonic balance analysis for electromagnetic (EM) energy harvesting," IEEE Antennas Wireless Propag. Lett., vol. 9, pp. 444-446, 2010.

[39] J. Y. Park, S. M. Han, and T. Itoh, "A rectenna design with harmonic-rejecting circular-sector antenna," IEEE Antennas Wireless Propag. Lett., vol. 5, no. 1, pp. 52-54, 2004. 
[40] M. Ali, G. Yang, and R. Dougal, "Miniature circularly polarized rectenna with reduced out of band harmonics," IEEE Antennas Wire-less Propag. Lett., vol. 5, no. 1, pp. 107-110, 2006.

[41] "Self-aligning nano particles for transparent electronics," [Online]. Available:

http://www.cimananotech.com 
TABLE I

DIMENSIONS (MM) OF THE ANTENNA GEOMETRY IN FIG. 1

\begin{tabular}{|c|c|c|c|c|c|c|}
\hline Parameters & $\mathrm{L}$ & $\mathrm{W}$ & $\mathrm{L}_{1}$ & $\mathrm{~L}_{2}$ & $\mathrm{~W}_{1}$ & $\mathrm{~g}$ \\
\hline Free space & 33.5 & 17.0 & 11.3 & 15.8 & 7.5 & 0.1 \\
\hline 2mm Glass & 35.1 & 17.5 & 10.6 & 15.8 & 7.82 & 0.23 \\
\hline $\begin{array}{c}\text { Sandwich } \\
\text { 2mm Glass/Solar }\end{array}$ & 44.6 & 25.5 & 7.86 & 19.3 & 11.04 & $\mathbf{0 . 2 8}$ \\
\hline
\end{tabular}

TABLE II

OUTPUT VOLTAGE OF RECTIFIER FOR 30-dBm INPUT POWER

\begin{tabular}{|c|c|c|c|c|c|c|c|c|c|c|c|}
\hline $\begin{array}{c}\text { Frequency } \\
\text { (GHz) }\end{array}$ & 2.50 & 2.51 & 2.52 & 2.53 & 2.54 & 2.55 & 2.56 & 2.57 & 2.58 & 2.59 & 2.60 \\
\hline Voltage (mV) & 14.8 & 16.5 & 17.8 & 19.6 & 23.6 & 25.6 & 22.4 & 20.9 & 18.2 & 14.4 & 10.5 \\
\hline
\end{tabular}

TABLE 3

OUTPUTVOLTAGE OF RECTENNA FOR $30 \mathrm{dBm}$ INPUT POWER

\begin{tabular}{|c|c|c|c|c|c|c|c|c|c|c|c|c|}
\hline \multirow{2}{*}{$50 \Omega$} & Frequency (GHz) & 2.50 & 2.51 & 2.52 & 2.53 & 2.54 & 2.55 & 2.56 & 2.57 & 2.58 & 2.59 & 2.60 \\
\cline { 2 - 12 } & Voltage (mV) & 7.1 & 8.4 & 8.8 & 9.4 & 9.2 & 9.2 & 9.1 & 8.6 & 7.5 & 7.4 & 7.2 \\
\hline \multirow{2}{*}{$100 \Omega$} & Frequency (GHz) & 2.50 & 2.51 & 2.52 & 2.53 & 2.54 & 2.55 & 2.56 & 2.57 & 2.58 & 2.59 & 2.60 \\
\cline { 2 - 11 } & Voltage (mV) & 17.8 & 18 & 17 & 16.7 & 15.3 & 16 & 16.4 & 17 & 16 & 15.4 & 14.2 \\
\hline
\end{tabular}

TABLE 4

OUTPUT VOLTAGE OF CSTA SOLAR MODULE (mV)

\begin{tabular}{|l|c|c|c|}
\cline { 2 - 3 } \multicolumn{1}{c|}{} & \multicolumn{2}{c|}{ Ambient Light } & \multirow{2}{*}{ Sun } \\
\cline { 2 - 3 } \multicolumn{1}{c|}{} & VNA (Off) & VNA (On) & \\
\hline Solar only & 102.9 & - & 4.5 \\
\hline CTSA + Solar & 76.1 & 74.4 & 4.5 \\
\hline Glass+CTSA+Solar & 68.6 & 68.7 & 4.7 \\
\hline Glass+Solar & 93.2 & - & 4.5 \\
\hline
\end{tabular}


Fig. 1. (a) Geometry of CTSA and (b) prototype of CTSA.

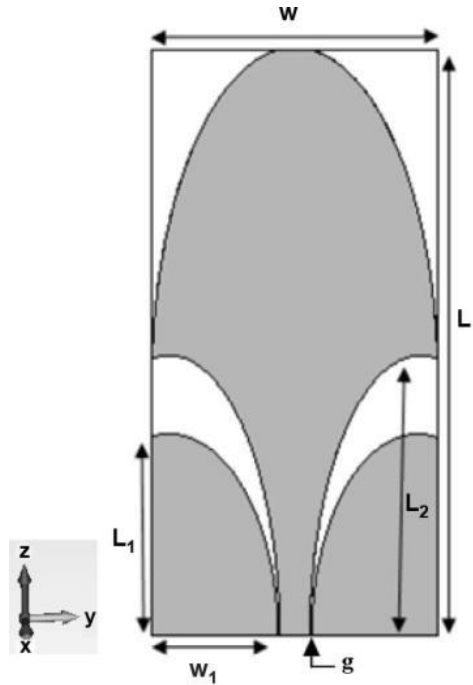

(a)

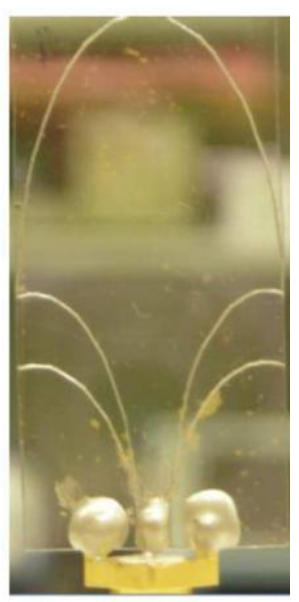

(b)

Fig. 2. Structural view of CTSA sandwiched between glass superstrate and a-Si solar panel.

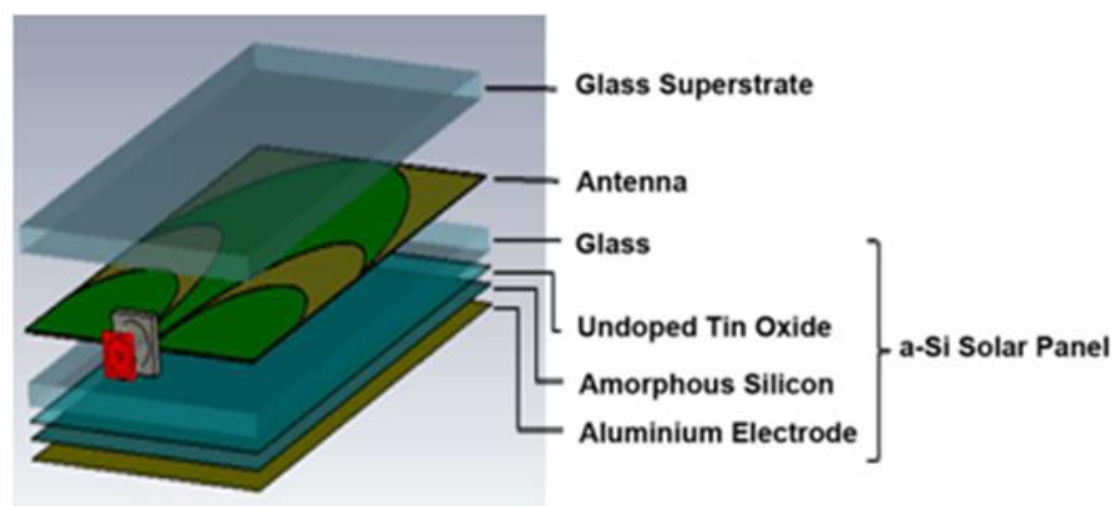

Fig. 3. Structure of a-Si solar cell.

\begin{tabular}{|c|c}
\hline Glass Substrate & \multirow{1}{*}{$1.8 \mathrm{~mm}$} \\
\cline { 1 - 1 } Tin Oxide (Transparent Electrode) & $70 \mathrm{~nm}$ \\
\cline { 1 - 1 } p-type layer & $10 \mathrm{~nm}$ \\
\cline { 1 - 1 } Amorphous Silicon & $600 \mathrm{~nm}$ \\
\cline { 1 - 1 } n-type layer & $1000 \mathrm{~nm}$ \\
\cline { 1 - 1 } Aluminium Electrode &
\end{tabular}


Fig. 4. Schematic for voltage doubler.

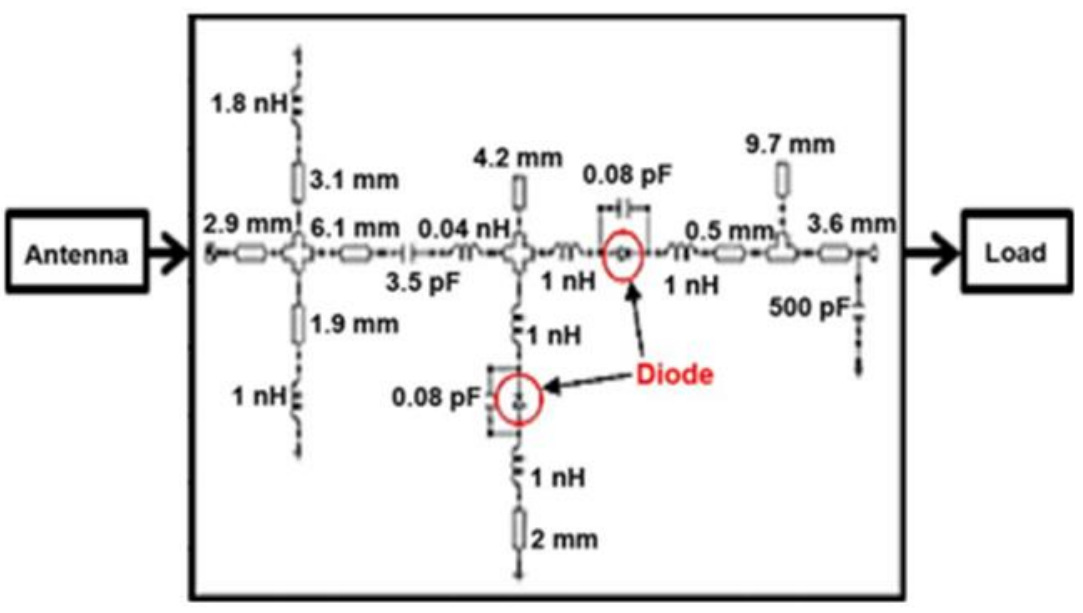

Fig. 5. Prototype of CTSA-rectifier module.

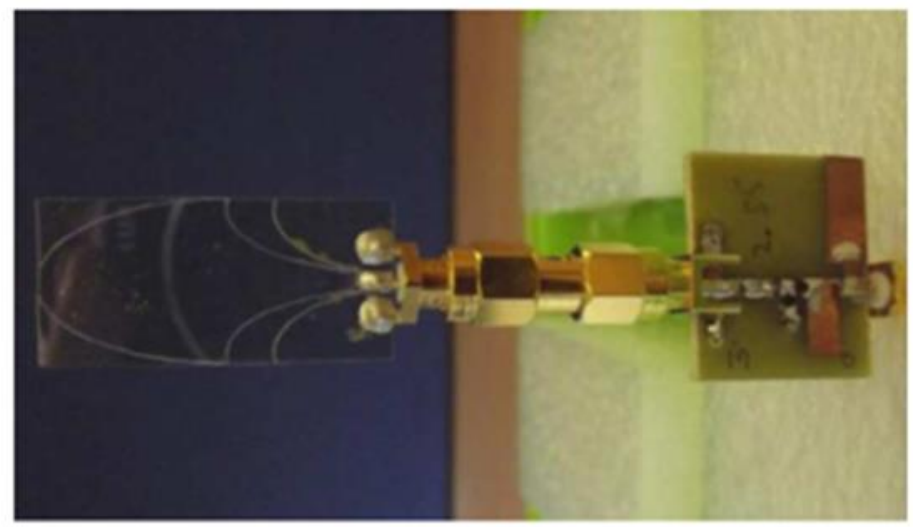

Fig. 6. Measurement setup for the CTSA rectenna (diagrammatic representation).

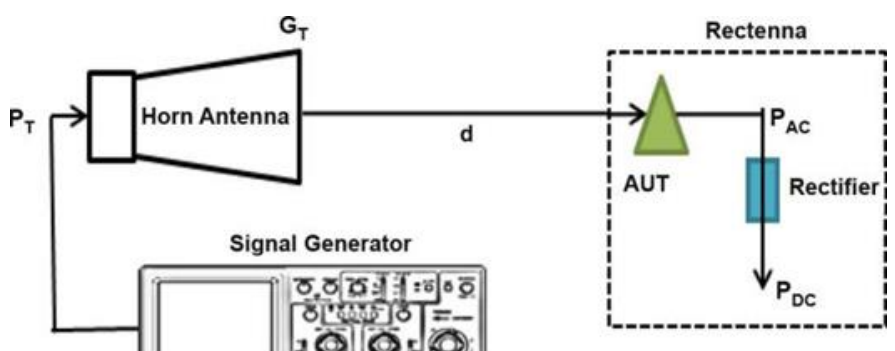


Fig. 7. Return losses of rectifier and rectenna.

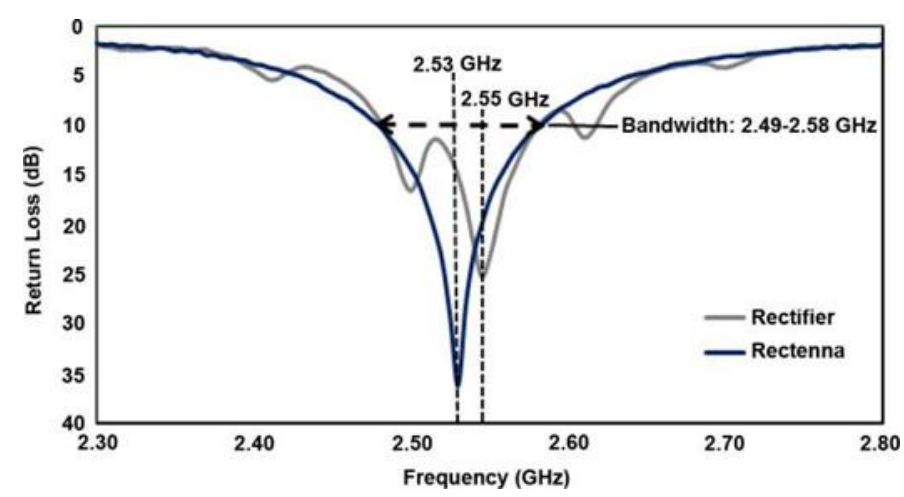

Fig. 8. Simulated and measured return loss of CTSA in free space, on solar panel only, and sandwiched between solar panel and glass.

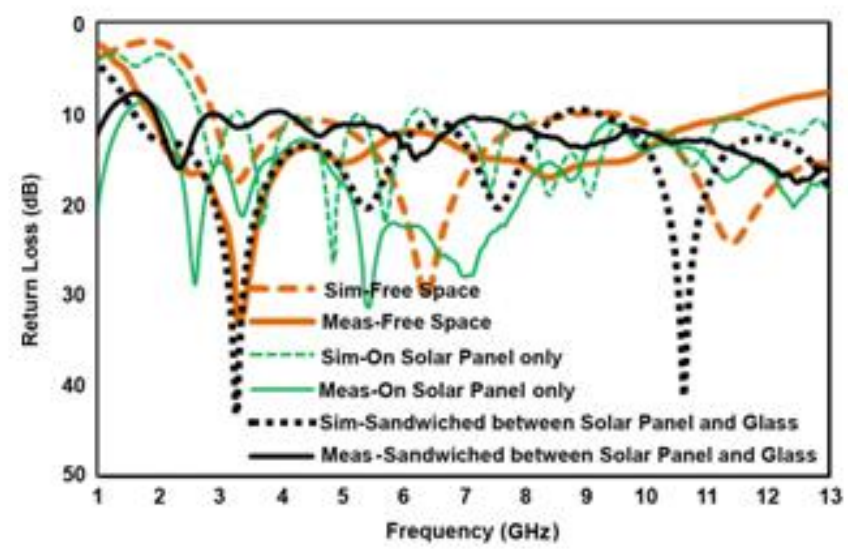

Fig. 9. Overall simulated and measured gains of CTSA in free space, on solar panel only, and sandwiched between solar panel and glass.

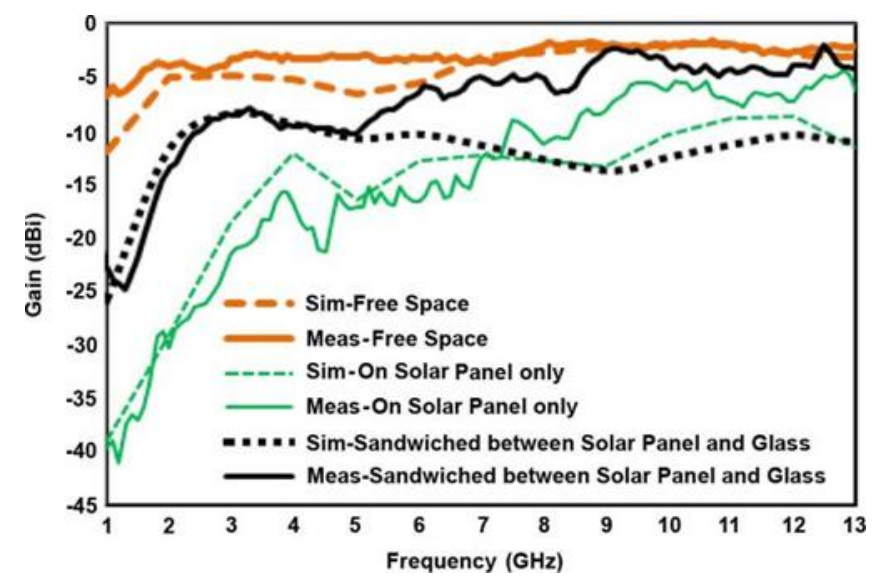


Fig. 10. Measured radiation patterns for CTSA in free space, on solar panel and sandwiched between a-Si solar panel and 2-mm glass at 3, 5, 7, and $10 \mathrm{GHz}$.
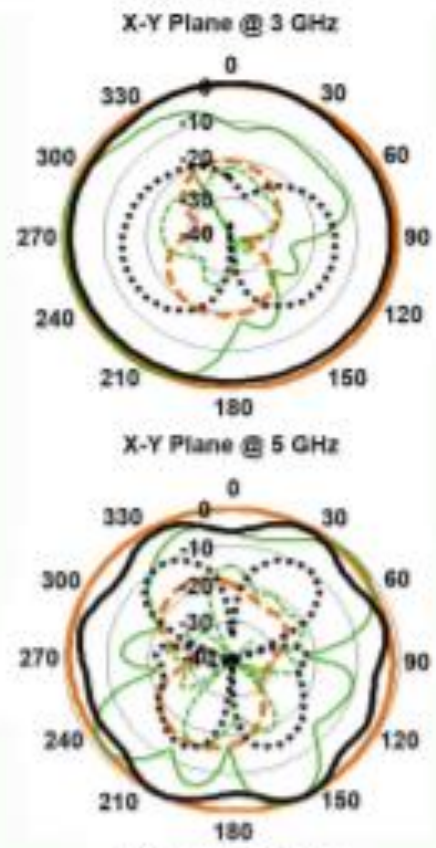

$X-Y$ Plane $87 \mathrm{GHz}$

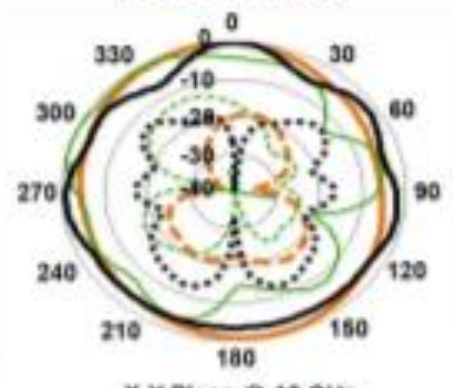

X.Y Ptane af 10 Gre

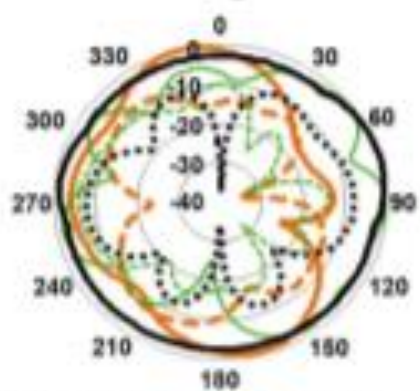

CoPolar free Space

Co Polar-On solar Panel only

$--\times$ Xolar-free space

X Polar-On Solar Panel enty

Co Polar - Sandwiched between Solar Panel and Glass

..... X Potar - Sandaiched between Solar Panei and Olass 
Fig. 11. Measured output power of CTSA rectenna versus input power on a $50-\Omega$ and $100-\Omega$ load at 2.51 and $2.53 \mathrm{GHz}$ in free space.

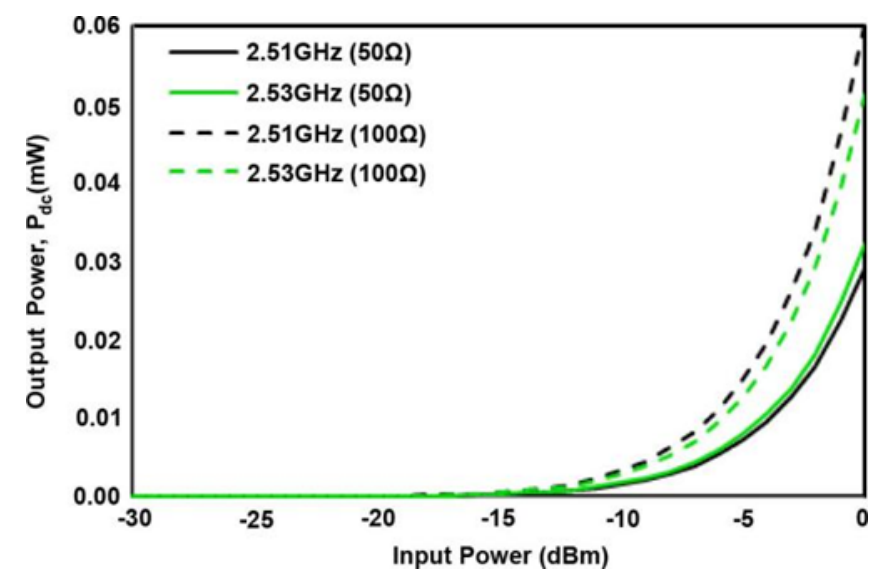

Fig. 12. Measured rectenna system efficiencies at 2.51 and $2.53 \mathrm{GHz}$ at a $50-\Omega$ and $100-\Omega$ loads in free space.

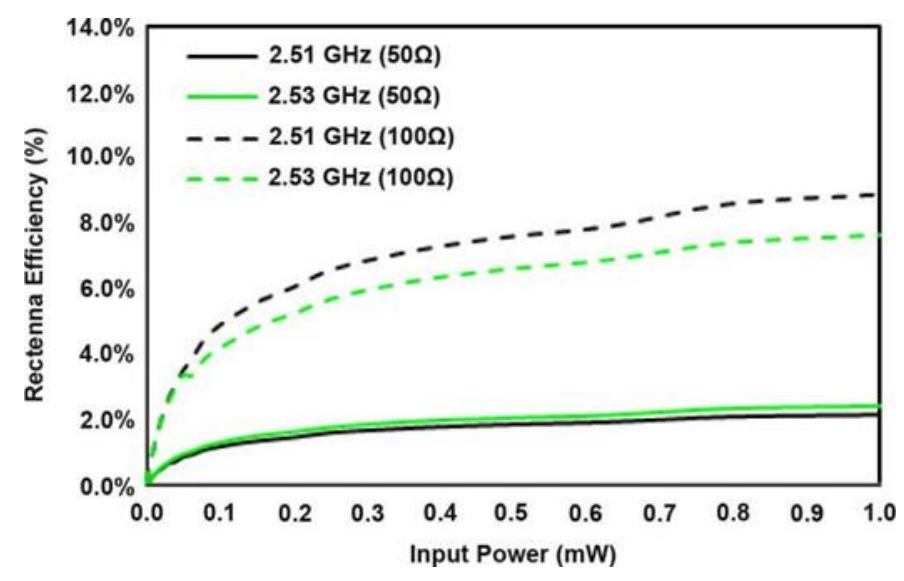

Fig. 13. Simulated and measured return loss of rectenna on glass. 


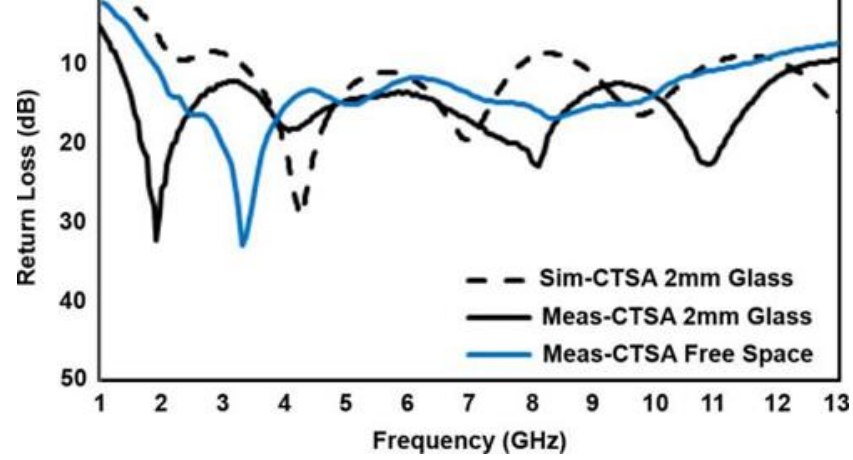

Fig. 14. Output voltage optimum at $2.57 \mathrm{GHz}$ for $50-\Omega$ and $100-\Omega$ loads at $10-\mathrm{dBm}$ input on 2-mm glass. Free-space results given for comparison.

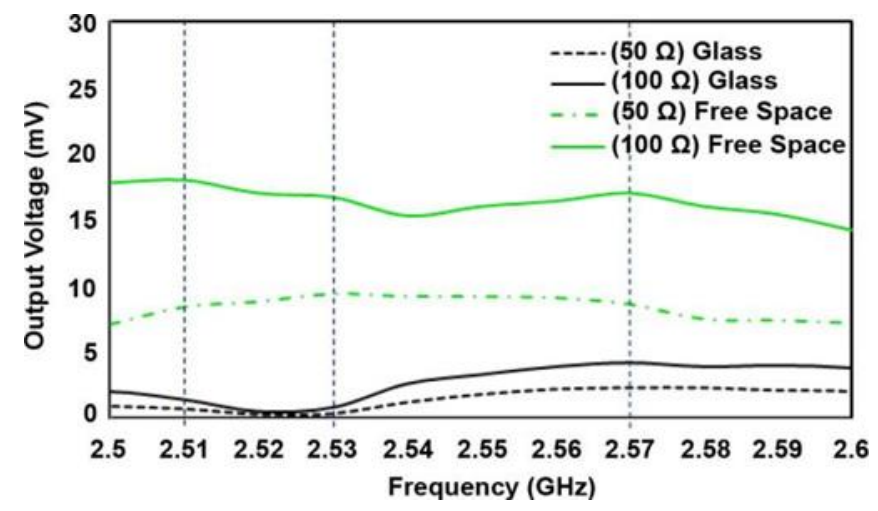

Fig. 15. Measured gain of CTSA on 2-mm glass substrate.

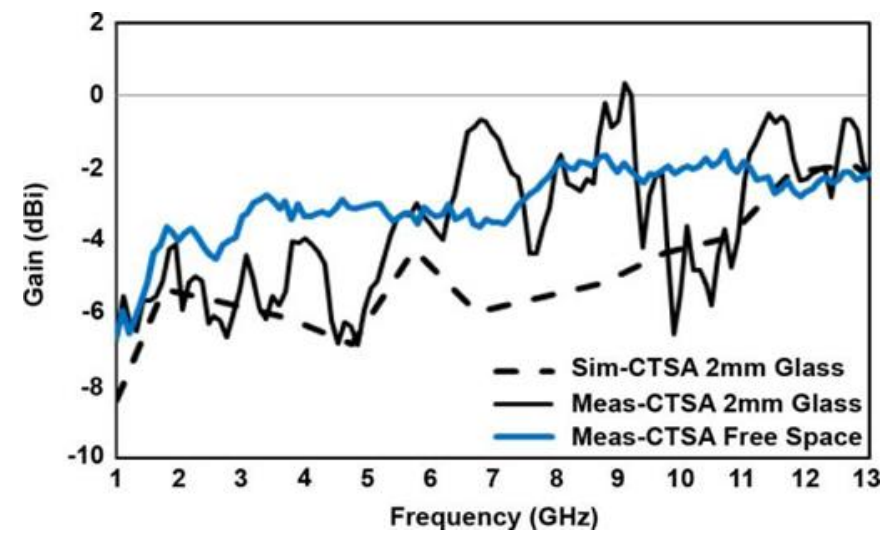


Fig. 16. Fidelity factor of the CTSA: (a) free space (b) on 2-mm glass substrate (c) sandwiched between solar panel and 2-mm glass.

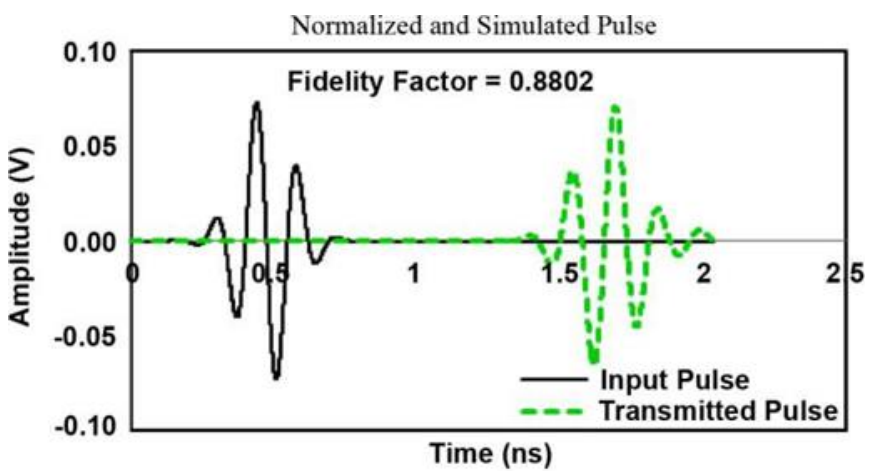

(a)

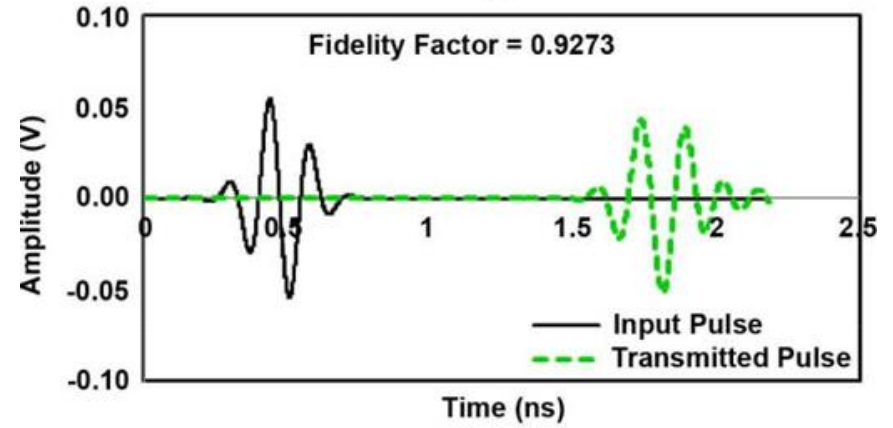

(b)

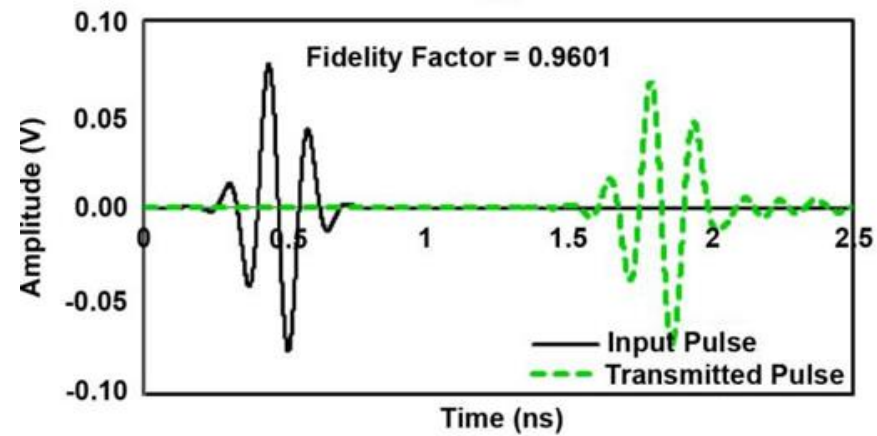

(c) 